\title{
The Thy-Angle and $g$-Angle in a Quasi-Inner Product Space
}

\author{
PAVle M. MiličIĆ
}

\begin{abstract}
In this note we prove that in a so-called quasi-inner product spaces, introduced a new angle (Thy-angle) and the so-called $g$ angle (previously defined) have many common characteristics. Important statements about parallelograms that apply to the Euclidean angles in the Euclidean space are also valid for the angles in a q.i.p. space (see Theorem 1).
\end{abstract}

\section{INTRODUCTION}

Let $(X,\|\cdot\|)$ be a seminormed space. Based an idea of I. Singer [5], Volker Thürey in [6] introduced a new concept of an angle between elements $x$ and $y$ of $X \backslash\{0\}$, so-called Thy-angle $\left(\angle_{T h y}(x, y)\right)$, as follows:

$$
\angle_{T h y}(x, y):=\arccos \left[\frac{1}{4}\left(\left\|\frac{x}{\|x\|}+\frac{y}{\|y\|}\right\|^{2}-\left\|\frac{x}{\|x\|}-\frac{y}{\|y\|}\right\|^{2}\right)\right] .
$$

This new angle corresponds with the Euclidean angle in the case that $(X,\|\cdot\|)$ already is an inner product space. In the real normed space $(X,\|\cdot\|)$, $\operatorname{dim} X>1, x, y \neq 0$, for this angle we have the properties:

1) $\angle_{T h y}$ is a continuous surjective function from $[X \backslash\{0\}]^{2}$ to $[0, \pi]$,

2) $\angle_{T h y}(x, x)=0$,

3) $\angle_{T h y}(-x, x)=\pi$,

4) $\angle_{T h y}(x, y)=\angle_{T h y}(y, x)$,

5) $\angle_{T h y}(r x, s y)=\angle_{T h y}(x, y), r, s>0$,

6) $\angle_{T h y}(-x,-y)=\angle_{T h y}(x, y)$,

7) $\angle_{T h y}(x, y)+\angle_{T h y}(-x, y)=\pi$.

With this angle we observe here is another angle was previously defined, which we now define.

2000 Mathematics Subject Classification. Primary: 46B20, 46C15, 51K05.

Key words and phrases. Quasi-Inner Product Space, Thy-Angle, g-Angle. 
It is well known that in a real smooth normed space $(X,\|\cdot\|)$, always exists the functional

$$
g(x, y):=\|x\| \lim _{t \rightarrow 0} \frac{\|x+t y\|-\|x\|}{t} \quad(x, y \in X) .
$$

(see [1]).

This functional is linear in second argument and it has the following properties:

$$
\begin{aligned}
g(r x, y)) & =r g(x, y), & & g(x, x)=\|x\|^{2}, \\
|g(x, y)| & \leqslant\|x\|\|y\|, & & (x, y \in X ; r \in R) .
\end{aligned}
$$

In an arbitrary normed space, we are in [2] define another angle, so-called $g$-angle with

$$
\angle_{g}(x, y):=\arccos \frac{g(x, y)+g(y, x)}{2\|x\|\|y\|}, \quad(x, y \in X \backslash\{0\}),
$$

and the so-called $g$-orthogonality vectors with

$$
x \perp_{g} y \Leftrightarrow g(x, y)+g(y, x)=0, \quad(x, y \in X \backslash\{0\} .
$$

Let us mention there that the so-called Pythagorean orthogonality vectors defines

$$
x \perp_{P} y \Leftrightarrow\|x\|^{2}+\|y\|^{2}=\|x+y\|^{2}, \quad(x, y \in X \backslash\{0\}) .
$$

Also note that known the Singer orthogonality can be defined with

$$
x \perp_{S} y \Leftrightarrow \angle_{T h y}(x, y)=\frac{\pi}{2} .
$$

A normed space $(X,\|\cdot\|)$ of property

$$
\|x+y\|^{4}-\|x-y\|^{4}=8\left[\|x\|^{2} g(x, y)+\|y\|^{2} g(y, x)\right], \quad(x, y \in X)
$$

we call a quasi-inner product space (q.i.p space) (see [3]).

The space of sequences $l^{4}$ is a q.i.p. space

$$
\left.\left(x=\left(x_{k}\right), \quad y=\left(y_{k}\right) \in l^{4}, \quad g(x, y)=\|x\|^{-2} \sum_{k}\left|x_{k}\right|^{3}\left(\operatorname{sgn} x_{k}\right) y_{k}\right)\right),
$$

but $l^{1}$ is not a q.i.p. space (see [3]).

In [4] we have proved that, in a q.i.p. space

$$
x \perp_{g} y \Leftrightarrow x \perp_{S} y \quad(x, y \in X \backslash\{0\}) .
$$

Having regard to the equality (5) comparing the definition (1) and (4) we conclude that there is a direct link between $g$-angle and Thy-angle. 
Namely, for $x, y \neq 0$, if instead $x$ it takes place $\frac{x}{\|x\|}$ and instead $y$ take $\frac{y}{\|y\|}$, from (5) we get

$$
\begin{aligned}
& \frac{1}{4}\left[\left\|\frac{x}{\|x\|}+\frac{y}{\|y\|}\right\|^{2}+\left\|\frac{x}{\|x\|}-\frac{y}{\|y\|}\right\|^{2}\right] . \\
& \cdot \frac{1}{4}\left[\left\|\frac{x}{\|x\|}+\frac{y}{\|y\|}\right\|^{2}-\left\|\frac{x}{\|x\|}-\frac{y}{\|y\|}\right\|^{2}\right]= \\
& =\frac{g(x, y)+g(y, x)}{2\|x\|\|y\|} .
\end{aligned}
$$

From this equality and (3) we conclude that

$$
\begin{aligned}
-1 \leq & \frac{1}{4}\left[\left\|\frac{x}{\|x\|}+\frac{y}{\|y\|}\right\|^{2}+\left\|\frac{x}{\|x\|}-\frac{y}{\|y\|}\right\|^{2}\right] . \\
& \cdot \frac{1}{4}\left[\left\|\frac{x}{\|x\|}+\frac{y}{\|y\|}\right\|^{2}-\left\|\frac{x}{\|x\|}-\frac{y}{\|y\|}\right\|^{2}\right] \leqslant 1 .
\end{aligned}
$$

This means that in a q.i.p. spaces $X$ can be define another angle between vectors $x, y \in X \backslash\{0\}$ with

$$
\angle(x, y):=\arccos \frac{1}{16}\left[\left\|\frac{x}{\|x\|}+\frac{y}{\|y\|}\right\|^{4}-\left\|\frac{x}{\|x\|}-\frac{y}{\|y\|}\right\|^{4}\right] .
$$

In fact in a q.i.p. space, this angle is equal to $g$-angle.

Knowing that

$$
g(r x, s y)=g(x, y) \quad(r, s \neq 0)
$$

it can be seen instead $\angle(x, y)$ the angle $\angle(u, v)$, where

$$
u=\frac{x}{\|x\|}, v=\frac{y}{\|y\|} \in S(X)
$$

$(S(X)$ is the unit sphere of $X)$.

Then (6) becomes

(8) $\frac{1}{4}\left[\|u+v\|^{2}+\|u-v\|^{2}\right] \cdot \frac{1}{4}\left[\|u+v\|^{2}-\|u-v\|^{2}\right]=\frac{g(u, v)+g(v, u)}{2}$.

Checking more

$$
\begin{aligned}
k & =\frac{1}{4}\left[\|u+v\|^{2}+\|u-v\|^{2}\right], \\
b & =\frac{1}{4}\left[\|u+v\|^{2}-\|u-v\|^{2}\right], \\
a & =\frac{g(u, v)+g(v, u)}{2}
\end{aligned}
$$

get

$$
k b=a,
$$


i.e., for all $u, v \in S(X), \arccos k b=\arccos a$.

This means that, for $x, y \in X \backslash\{0\}$

$$
\angle(x, y)=\angle_{g}(x, y) .
$$

Although $\angle_{g}(u, v)$ and $\angle_{T h y}(u, v)$ are two mutually different functional they, in a q.i.p. space, have many common characteristics.

Modeled in terms of Euclidean geometry, we adopt the following terminology in normed spaces.

From now on we assume that points $0, x, y$ are the vertices of the triangle $(0, x, y)$ and points $0, x, y, x+y$ are the vertices of the parallelogram $(0, x, y, x+y)$. The numbers $\|x+y\|,\|x-y\|$ are the lengths of diagonal of this parallelogram. If $\|x\|=\|y\|$, we say that this parallelogram is a romb, and if $x \perp_{\rho} y$ we say that the parallelogram $(0, x, y, x+y)$ is a $\rho$ - rectangle.

\section{Main Results}

Justification for introducing these angles in the normed spaces show, among other things, the following Theorem 1.

Theorem 1. Let $X$ be a q.i.p. space. The following statements are true:

a) The g-angle has properties 1)-7), similar to the Thy-angle;

b) The lengths of diagonals parallelogram $(0, x, y, x+y)$ are equal if and only if this parallelogram is Thy-rectangle, i.e. $x \perp_{S} y$ or $\angle_{\text {Thy }}(x, y)=$ $\pi / 2$;

c) The diagonals of the romb $(0, x, y, x+y)$ are Thy-orthogonal, i.e. $(x-y) \perp_{S}(x+y)$

d) The parallelogram $(0, x, y, x+y)$ is a Thy-quadrangle if and only if its lengths of the diagonals are equal and the diagonals are Thyorthogonal.

Proof. Using the properties (3) of $g$-functional easy to check these properties 1)-7) are valid for the $g$-angle.

For evidence statements b)-d) to use gender The-orthogonality of the $g$ orthogonality $\left(\perp_{T h y}=\perp_{g}\right)$ since the $g$-orthogonality assertion is proved in [4].

Following two theorems show the relationship of these two angles depending on the vectors $u, v \in S(X)$.

Theorem 2. Let $X$ be a q.i.p. space. The following statements are true:

1. For all $u, v \in S(X)$ it is $\operatorname{sgn} a=\operatorname{sgn} b$, i.e., $\operatorname{sgn} \angle_{g}(u, v)=\operatorname{sgn} \angle_{T h y}(u, v)$,

2. $\angle_{g}(u, v)=\angle_{T h y}(u, v) \Leftrightarrow(u+v) \perp_{P}(u-v) \vee u \perp_{g} v$.

Proof.

1. Since the $k>0$ from (9) we get $\operatorname{sgn} a=\operatorname{sgn} b$. 
2. According to the definitions (1) and (4) we have (9) so

$$
\angle_{g}(u, v)=\angle_{T h y}(u, v) \Leftrightarrow k=1 \vee\|u+v\|=\|u-v\| .
$$

If $k=1$ then

$a=b \wedge\|u+v\|^{2}+\|u-v\|^{2}=4=\|(u+v)+(u-v)\|^{2} \Leftrightarrow\left(u+v \perp_{P}(u-v)\right.$.

Since $k>0$ it is

$$
\|u+v\|=\|u-v\| \Leftrightarrow a=b=0 \Leftrightarrow \angle_{g}(u, v)=\angle_{T h y}(u, v) .
$$

The interrelation of angles $\angle_{g}(u, v)$ and $\angle_{T h y}(u, v)$ depends on the relationship between length of diagonals of a parallelogram $(0, u, v, u+v)$.

Theorem 3. Let $X$ be a q.i.p. space. The following assertion are valid:

1. If $\|u-v\|<\|u+v\|$ then

$$
\begin{array}{lll}
\|u+v\|^{2}+\|u-v\|^{2}>4 & \Rightarrow & \angle_{g}(u, v)<\angle_{T h y}(u, v), \\
\|u+v\|^{2}+\|u-v\|^{2}<4 & \Rightarrow & \angle_{g}(u, v)>\angle_{T h y}(u, v) .
\end{array}
$$

2. If $\|u-v\|>\|u+v\|$ then

$$
\begin{array}{lll}
\|u+v\|^{2}+\|u-v\|^{2}>4 & \Rightarrow & \angle_{g}(u, v)>\angle_{T h y}(u, v), \\
\|u+v\|^{2}+\|u-v\|^{2}<4 & \Rightarrow & \angle_{g}(u, v)<\angle_{T h y}(u, v) .
\end{array}
$$

Proof.

1. Since the $k>1$ and $b>0$ according to (9) we have

$$
a=k b>b \text {, so } \arccos a<\arccos b \text {, i.e., } \angle_{g}(u, v)<\angle_{T h y}(u, v) \text {. }
$$

If $k<1$ and $b>0$ then $a=k b<b$ so $\arccos a>\arccos b$, i.e., $\angle_{g}(u, v)>\angle_{T h y}(u, v)$.

2. Since $k>1$ and $b<0$ get $a=k b<b \Rightarrow a<b$, so

$$
\begin{gathered}
\arccos a>\arccos b \Leftrightarrow \angle_{g}(u, v)>\angle_{T h y}(u, v) . \\
k<1 \wedge b<0 \Rightarrow a=k b>b \Rightarrow a>b \Leftrightarrow \\
\arccos a<\arccos b \Leftrightarrow \angle_{g}(u, v) \quad<\quad \angle_{T h y}(u, v) .
\end{gathered}
$$

\section{REFERENCES}

[1] P.M. Miličić, Sur le produit scalaire géneralisé, Mat. Vesnik, (25)10) (1973), 325-329.

[2] P.M. Miličić, Sur la g-angle dans un espace normé, Mat. Vesnik, 45 (1993), 69-77.

[3] P.M. Miličić, A generalization of the parallelogram equality in normed spaces, Jour. Math. Kyoto Univ (JMKYAZ), 38(1) (1998), 71-75.

[4] P.M. Miličić, On the quasi-inne product spaces, Matematički Bilten, 22(XLVIII) (1998), 19-30. 
[5] I. Singer, Unghiuri Abstracte si Functii Trigonometrice in Spatii Banach, Buliten Stintefic, Sectia de Stiinte Matematice si Fisice, Academia Republicii Populare Romine, 9 (1957), 29-42.

[6] V. Thürey, Angles and Polar Cordinates in Real Normed Spaces, arXiv:0902.2731v2.

Pavle M. Milićić

Faculty of Mathematics

UNIVERSITY OF BELGRADE

STUDENTSKI TRG 16

11000 BeLgRADE

SERBIA

E-mail address: pavle.milicic@gmail.com 